\title{
ESTUDO DO NITROGÊNIO NA COMBUSTÃO DO GÁS NATURAL
}

Leonardo Paes Rangel*

Escola Politécnica, Universidade de Pernambuco, Rua Benfica, 455, 50750-410 Recife - PE

Pedro Maurício Büchler

Departamento de Engenharia Química, Escola Politécnica, Universidade de São Paulo, Av. Prof. Luciano Gualberto, 380 ,

Trav. 3, 05508-900 São Paulo - SP

Recebido em 7/6/04; aceito em 28/3/05; publicado na web em 8/9/05

\begin{abstract}
STUDY OF NITROGEN IN THE COMBUSTION OF NATURAL GAS. Nitrogen content in natural gas was studied in experimental and computational investigations to identify its influence on the emission level of exhaust gases from combustion facilities. Changes in natural gas composition with different $\mathrm{N}_{2}$ concentrations may result from introducing a new source gas into the system. An industrial burner fired at $75 \mathrm{~kW}$, housed in a laboratory-scale furnace, was employed for runs where the natural gas $/ \mathrm{N}_{2}$ proportion was varied. The exhaust and in-furnace measurements of temperature and gas concentrations were performed for different combustion scenarios, varying $\mathrm{N}_{2}$ content from 1-10\%v. Results have shown that the contamination of natural gas with nitrogen reduced the peak flame temperature, the concentration of unstable species, the $\mathrm{NO}_{\mathrm{x}}$ emission level and the heat transfer rate to the furnace walls, resulting from the recombination reactions.
\end{abstract}

Keywords: natural gas; combustion; nitrogen.

\section{INTRODUÇÃO}

Até 1998 o gás natural teve uma participação coajuvante na matriz energética brasileira de apenas $2,7 \%$. O aumento do seu consumo, através da importação de países vizinhos (Argentina e Bolívia), está causando uma diversificação na sua matriz, pois não existem, até o presente momento, grandes reservas de gás no país. De acordo com a projeção de dados de consumo, a participação do gás natural atual (2004) é de $9 \%$ e será cerca de $12 \%$ até $2010^{1}$.

Após a inclusão das novas diretrizes energéticas no Brasil, o gás natural será utilizado também para geração de energia elétrica com 49 novas termelétricas sendo construídas em todo o país. Quase todas deverão ser operadas à gás natural. Com a finalização da construção do Gasbol (gasoduto entre a Bolívia e o Brasil, com comprimento de $3150 \mathrm{~km}$ ) em 1999, o Brasil interligou os estados do Centro-Oeste, Sudeste e Sul, implementando um passo decisivo no suprimento de energia para as regiões mais industrializadas do país.

Ciente do consumo crescente do gás natural no mundo, o Brasil busca se beneficiar de uma alternativa mais eficiente para o processo de combustão industrial. Assim, grandes volumes de gás natural serão empregados em diferentes segmentos da indústria, como metalúrgica, cerâmica e têxtil, onde restrições ambientais têm aumentado particularmente nos últimos anos.

Tanto do ponto de vista energético como ambiental, as tecnologias relacionadas ao gás natural apresentam maior eficiência, principalmente quando comparadas com outros combustívies fósseis. Assim, é relevante a atenção sobre as técnicas de modificação no sistema de combustão, como o uso de queimadores de baixa emissão de $\mathrm{NO}_{\mathrm{x}}$.

O monóxido de nitrogênio (NO) e o dióxido de nitrogênio $\left(\mathrm{NO}_{2}\right)$ são os constituintes do $\mathrm{NO}_{\mathrm{x}}$. Este faz parte das reações de combustão de combustíveis fósseis e contribui para a poluição atmosférica. $\mathrm{O} \mathrm{NO}_{\mathrm{x}}$ foi identificado como o principal agente da disposição pluviométrica ácida (chuva ácida), que está relacionada com vári-

*e-mail: lprangel@gmail.com os danos ambientais e à saúde humana, incluindo acidificação de lagos e correntes, impactos em solos de florestas e a corrosão acelerada de edifícios ${ }^{2,3}$.

O gás natural é uma mistura de vários gases combustíveis, mas algumas substâncias inertes, como $\mathrm{CO}_{2}$ e $\mathrm{N}_{2}$, estão sempre presentes. De forma geral, o gás natural apresenta uma maior proporção de $\mathrm{CH}_{4}$ e uma menor proporção composta por outros hidrocarbonetos, como alcanos e alcenos. Assim, o gás natural não possui uma composição química fixa e a proporção de hidrocarbonetos e gases não-hidrocarbonetos pode variar amplamente, dependendo de sua origem. O gás natural boliviano, por ex., contém cerca de $1 \% \mathrm{v}$ de $\mathrm{N}_{2}$, que ainda pode depender da contribuição de cada campo de extração na composição do gás vendido. Outras fontes apresentam quantidades elevadas de $\mathrm{N}_{2}$, como o campo de Groningen (Holanda) com $14 \% v^{4}$.

Este trabalho apresenta o efeito da variação de concentração de $\mathrm{N}_{2}$ na composição do gás natural, investigado em relação aos níveis de emissão de $\mathrm{NO}_{\mathrm{x}}$. Na prática, a concentração de $\mathrm{N}_{2}$ no gás natural pode afetar a combustão através dos seus vários mecanismos de reação. Os resultados desse trabalho permitirão delinear uma nova metodologia na utilização de combustíveis gasosos com diferentes concentrações de $\mathrm{N}_{2}$.

$\mathrm{O}$ trabalho foi dividido em duas partes: investigações teóricas e experimentais. As investigações experimentais foram conduzidas em uma fornalha de bancada. Um queimador comercial para gás pré-aquecido com capacidade térmica de $75 \mathrm{~kW}$ foi utilizado, permitindo a formação de chamas com temperaturas de até $1800 \mathrm{~K}$. A fundamentação das investigações teóricas foi estudada através de um modelo computacional do queimador em um código comercial de CFD ("Computational Fluid Dynamics") com pré-processador para cálculo da emissão de $\mathrm{NO}_{\mathrm{X}}{ }^{5}$.

\section{PARTE EXPERIMENTAL}

Os equipamentos utilizados na etapa experimental foram uma fornalha de bancada, queimador à gás natural com ar pré-aquecido 
opcional, sistema de amostragem para gases de exaustão intra-fornalha, conjunto de analisadores de gases $\left(\mathrm{NO}_{\mathrm{x}}, \mathrm{CO}_{2}, \mathrm{CO}\right.$ e $\left.\mathrm{O}_{2}\right)$ e unidade computadorizada para aquisição de dados.

O objeto do experimento foi um queimador comercial de gás natural com ar estagiado e pré-aquecido, com capacidade térmica máxima de $100 \mathrm{~kW}$, projetado e fabricado por Stordy Combustion Engineering Ltd. (Inglaterra). A estratégia de queima foi configurada com pré-mistura ar/combustível no tubo externo e ar estagiado em alta velocidade através de um tubo de carbeto de silício central (Figura 1).

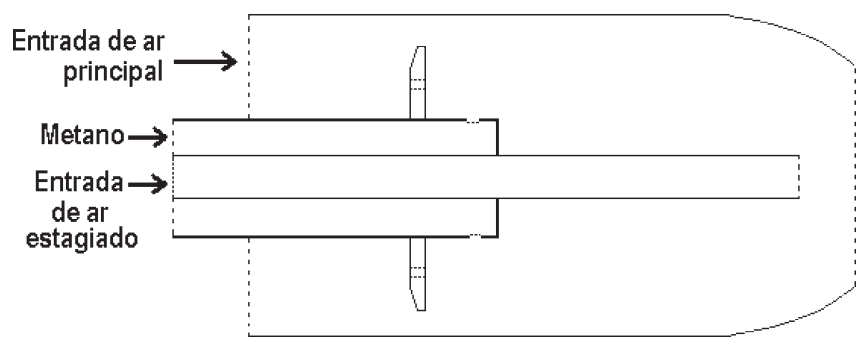

Figura 1. Diagrama 2D do queimador

A chama foi considerada estável sob condições de operação com deficiência de ar. $\mathrm{O}$ ar estagiado foi injetado na direção do eixo central, próximo a saída do queimador. Assim, a combustão foi operada na zona rica de combustível e concluída por um jato central de ar, a $10 \mathrm{~cm}$ da câmara de combustão. Neste trabalho foi utilizado um pré-aquecedor elétrico de ar, pois a fornalha não contava com um sistema de recirculação de gás. As condições de operação típicas do queimador são mostradas na Tabela 1 .

Tabela 1. Condições de operação do queimador

\begin{tabular}{ll}
\hline Potência Térmica & $50-100 \mathrm{~kW}$ \\
Fluxo volumétrico de gás natural & $113-2261 \mathrm{~min}^{-1}(\mathrm{CNTP})$ \\
Fluxo volumétrico de ar & $1000-24001 \mathrm{~min}^{-1}$ (CNTP) \\
Temperatura de ar (pré-aquecido) & $25-1300^{\circ} \mathrm{C}$ \\
Temperatura média da parede & $900-1100{ }^{\circ} \mathrm{C}$ \\
Razão ar/combustível & $0,8-1,1$
\end{tabular}

Uma fornalha experimental (3,00 $\mathrm{m}$ de comprimento, $0,60 \mathrm{~m}$ de largura e $0,90 \mathrm{~m}$ de altura) foi revestida internamente com uma cerâmica refratária de aquecimento rápido e com uma camada de $0,025 \mathrm{~m}$ de fibras cerâmicas do tipo Triton. A fornalha foi cimentada externamente com uma camada de tijolos tipo MPK 110. Sua camada externa acomoda ainda $0,03 \mathrm{~m}$ de espessura de fibras minerais e uma lâmina de aço.

Os orifícios de amostragem foram dispostos a diferentes distâncias $(0,15 ; 0,45 ; 0,75 ; 1,05 ; 1,50 ; 2,10$ e 2,70 m) da parede interna da fornalha, considerando o lado da entrada do queimador. Sete furos passantes $(0,05 \mathrm{~m}$ de diâmetro) foram alocados na linha de simetria horizontal de uma das paredes laterais da fornalha, para coleta de amostras através de uma sonda isocinética de $1,50 \mathrm{~m}$ de comprimento. Do outro lado da fornalha, um furo de $0,19 \mathrm{~m}$ de diâmetro interno foi projetado para visualização da chama.

A zona de resfriamento inferior à água era de dimensões 0,60 x 0,18 x $1,80 \mathrm{~m}$. A pressão interna dos gases da fornalha foi monitorada durante a operação do queimador através de um manômetro acoplado a um sinal digital. As temperaturas da fornalha foram monitoradas através de dois termopares tipo $\mathrm{K}$, inseridos na parede interna da fornalha a uma distância de 0,90 e 2,10 m ao lado do queimador.

\section{COMBUSTÃO TURBULENTA EM COMPUTAÇÃO FLUIDODINÂMICA}

O modelo em computação fluidodinâmica (CFD) gerencia a entrada de dados do problema e adapta as sub-rotinas do módulo solver. As atividades do usuário no estágio de pré-processamento envolvem definição da geometria da região de interesse (domínio computacional mostrado na Figura 2), definição das propriedades do fluido, especificação das condições de contorno apropriadas e geração da malha para o sistema queimador-fornalha (Figura 3).

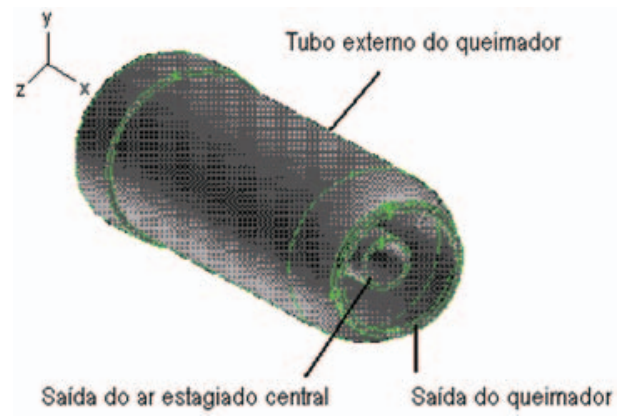

Figura 2. Modelo computacional do queimador em $3 D$

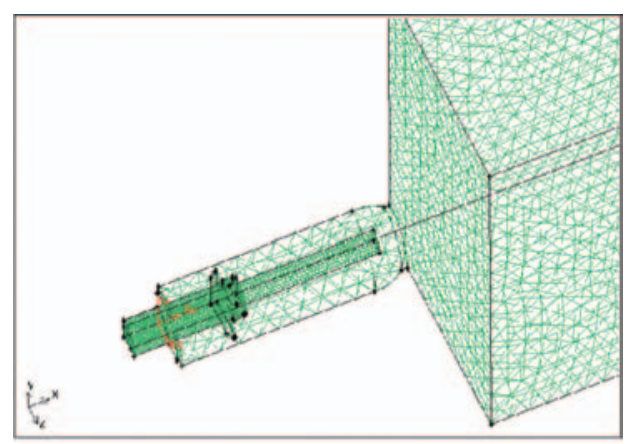

Figura 3. Domínio do sistema queimador-fornalha

O código comercial de computação fluidodinâmica FLUENT ${ }^{\text {TM }}$ resolve equações diferenciais parciais para conservação de massa, momento, energia e espécies químicas. De forma geral, essas equações podem ser mostradas por uma variável de conservação $\Phi$, como mostrado na Equação 1

$\frac{d}{d t}(\rho \Phi)+\frac{d}{d x_{i}}\left(\rho \mathrm{u}_{\mathrm{i}} \Phi\right)=\frac{d}{d x_{i}}\left[\Gamma \Phi \frac{d \Phi}{d x_{i}}\right]+\mathrm{S}_{\Phi}$

onde $\rho$ é densidade $\left[\mathrm{kg} \mathrm{m}^{-3}\right], u_{i}$ - velocidade da espécie química $i$, $t$ - tempo [s], $x$ - localização da espécie $i$ no fluxo [m], ГФ - gradiente de $\Phi$ no termo difusivo e $S_{\Phi}$ - termo de origem.

A fase de solução envolve a aproximação inicial das variáveis desconhecidas, ou discretização, onde a substituição de aproximações nas equações algébricas por meio da técnica computacional de interações gerou resultados com precisões cada vez maiores ${ }^{6}$.

O código comercial FLUENT ${ }^{\mathrm{TM}}$ permite o cálculo das espécies químicas em um módulo principal e um pós-processador trata os resultados gerados, de modo a calcular as concentrações dos poluentes sem interferir nos parâmetros iniciais do modelo. Este é o caso da formação de $\mathrm{NO}_{\mathrm{x}}$, que é acionada após o modelo realizar todos os cálculos para as variáveis intrínsicas (velocidade, temperatura, espécies químicas). 
Como o fluxo do sistema de combustão é turbulento, ocorrem flutuações temporais na temperatura e nas concentrações das espécies químicas, que possuem forte influência nas propriedades gerais da chama?.

A principal função do modelo de turbulência é fornecer uma expressão matemática para cálculo das propriedades do fluido em termos de "quantidade de fluxo". Para que os modelos de turbulência sejam úteis na abordagem de um código CFD, devem ter larga aplicabilidade, além de serem precisos e econômicos em termos computacionais. Para o presente trabalho foi usado o modelo de turbulência $\kappa-\varepsilon$, devido a suas características de precisão e baixo tempo computacional ${ }^{8}$. O modelo $\kappa-\varepsilon$ representa-se através de duas equações diferenciais, uma para a energia cinética $(\kappa)\left[\mathrm{m}^{2} \mathrm{~s}^{-2}\right]$ e outra para a taxa de dissipação da energia $(\varepsilon)\left[\mathrm{m}^{2} \mathrm{~s}^{-3}\right]$. Os vetores de Reynolds stress são assumidos como proporcionais ao gradiente de velocidade principal, com uma constante de proporcionalidade definida como viscosidade turbulenta $\left(\mu_{\mathrm{t}}\right)$.

A intensidade turbulenta (I) e o comprimento característico da chama ( $\ell$ ) são usados nas condições iniciais do modelo desenvolvido no código FLUENT ${ }^{\mathrm{TM}}$ para cálculo de $\varepsilon$ pela Equação 2. A taxa de dissipação de energia $\varepsilon$ é baseada na escala do comprimento turbulento $\ell$ relacionando-a com o tamanho dos grandes turbilhões que contém energia, presentes no fluxo turbulento. $\mathrm{O}$ valor inicial de $\kappa$ é calculado pela Equação 3.

$\varepsilon=C_{\mu}^{3 / 4} \frac{\sqrt{k^{3}}}{\ell}$

$\kappa=\frac{3}{2}(\bar{u} I)^{2}$

onde $\bar{u}$ é a magnitude da velocidade principal inicial $\left[\mathrm{m} \mathrm{s}^{-1}\right]$ e a $C_{\mu}$ é a constante de turbulência que vale 0,092 .

\section{MODELAGEM DAS REAÇÕES QUÍMICAS}

O código FLUENTTM permite a escolha entre três abordagens de mecanismos de reação química: formulação geral de taxa finita, frente pré-misturada e fração de mistura utilizando uma função probabilística de densidade (PDF - "probabilistic density function").

O conceito de fração de mistura foi utilizado para uma função PDF onde as equações de transporte das espécies químicas não são resolvidas individualmente, mas através de uma única equação, com variável escalar conservativa denominada fração de mistura (f). A função PDF considera os efeitos da turbulência através do modelo $\kappa-\varepsilon$ e as espécies químicas em equilíbrio ${ }^{5}$. Através da fração de mistura no modelo, é possível o cálculo de espécies intermediárias, efeitos de dissociação e uma relação entre efeitos turbulentos e reações químicas. A abordagem é computacionalmente eficiente, pois não requer uma solução para um grande número de equações de transporte (uma para cada espécie).

Os mecanismos de reações químicas para combustíveis hidrocarbonetos são complexos e a maioria das espécies quimica- mente reativas não possuem suas taxas de reação consideradas na análise de sistemas de combustão. O mecanismo resumido (com quatro etapas), aplicado no modelo deste trabalho permitiu uma razoável análise do comportamento da chama através das reações (R1) a (R4).

$$
\begin{aligned}
& \mathrm{CH}_{4}+\frac{1}{2} \mathrm{O}_{2} \stackrel{k_{1}}{\longrightarrow} \mathrm{CO}+2 \mathrm{H}_{2} \\
& \mathrm{CH}_{4}+\mathrm{H}_{2} \mathrm{O} \stackrel{k_{2}}{\longrightarrow} \mathrm{CO}+3 \mathrm{H}_{2} \\
& \mathrm{H}_{2}+\frac{1}{2} \mathrm{O}_{2} \stackrel{k_{3}}{\longrightarrow} \mathrm{H}_{2} \mathrm{O} \\
& \mathrm{CO}+\mathrm{H}_{2} \mathrm{O} \stackrel{k_{4}}{\longrightarrow} \mathrm{CO}_{2}+\mathrm{H}_{2}
\end{aligned}
$$

\section{MODELO QUÍMICO PARA FORMAÇÃO DE NO}

O NO pode ser formado através de três mecanismos químicos distintos: térmico, ativo e $\mathrm{N}_{2} \mathrm{O}$-intermediário, como mostrado na Tabela 2. A formação de $\mathrm{NO}$ pelo mecanismo $\mathrm{N}_{2} \mathrm{O}$-intermediário é importante apenas para condições de combustão pobre em combustível $(\phi<0,8)$ e sob temperaturas menores que $1200 \mathrm{~K}^{9}$. No presente trabalho esse mecanismo não apresentou importância, pois as simulações foram realizadas com altas temperaturas de chama e única condição de excesso de ar $(\phi=0,91)$. A taxa líquida da formação de NO térmico, principal contribuinte quando na combustão de gás natural, foi calculada pela Equação 4, onde as concentrações das espécies químicas estão em $\left[\mathrm{mol} \mathrm{m}^{-3}\right]$.

$$
\begin{aligned}
\frac{d[N O]_{t h}}{d t}=k_{1}[O]\left[N_{2}\right]+k_{2}[N]\left[O_{2}\right]+k_{3}[N][O H]- \\
k_{-1}[N O][N]-k_{-2}[N O][O]-k_{-3}[N O][H]
\end{aligned}
$$

Verificou-se que a taxa de formação do NO térmico é significante apenas para temperaturas maiores que $1000 \mathrm{~K}$, pois a reação com $\mathrm{o} \mathrm{N}_{2}$ do ar requer a quebra da ligação tripla a uma energia de dissociação de $941 \mathrm{~kJ} \mathrm{~mol}^{-1}{ }^{10}$.

A Equação 5 indica que a formação de NO varia com a concentração de $\mathrm{O}_{2}$ e, principalmente, com a temperatura. Bartok e Sarofim ${ }^{11}$ verificaram que a taxa de produção de NO aumenta em duas vezes a cada aumento de temperatura de $90 \mathrm{~K}$ após $2200 \mathrm{~K}$.

$\frac{d[N O]_{t h}}{d t}=2 k_{1}[O]\left[N_{2}\right] \frac{1-[N O]^{2} / K\left[O_{2}\right]\left[N_{2}\right]}{1+k_{-1}[N O] / k_{2}\left[O_{2}\right]+k_{3}[O H]}$

Para a resolução da Equação 5)foi necessário se conhecer as concentrações das espécies químicas $\mathrm{O}_{2}, \mathrm{~N}_{2}, \mathrm{O}$ e $\mathrm{OH}$. As concentrações de $\mathrm{O}_{2}$ e $\mathrm{N}_{2}$ foram medidas no sistema de combustão, porém as espécies instáveis $\mathrm{O}$ e $\mathrm{OH}$ foram apenas estimadas quando se assumiu um equilíbrio parcial, através das reações (R5) a (R8) ${ }^{7}$.

$\mathrm{H}+\mathrm{O}_{2} \stackrel{k_{5}}{\longrightarrow} \mathrm{OH}+\mathrm{O}$

Tabela 2. Mecanismos de reação para formação de $\mathrm{NO}_{\mathrm{x}}$

\begin{tabular}{lll}
\hline $\mathrm{NO}$ Térmico & $\mathrm{NO}$ Ativo & $\mathrm{N}_{2} \mathrm{O}$-intermediário \\
\hline $\mathrm{O}+\mathrm{N}_{2} \leftrightarrow \mathrm{NO}+\mathrm{N}$ & $\mathrm{CH}+\mathrm{N}_{2} \leftrightarrow \mathrm{HCN}+\mathrm{N}$ & $\mathrm{O}+\mathrm{N}_{2}+\mathrm{M} \leftrightarrow \mathrm{N}_{2} \mathrm{O}+\mathrm{M}$ \\
$\mathrm{N}+\mathrm{O}_{2} \leftrightarrow \mathrm{NO}+\mathrm{O}$ & $\mathrm{HCH}+\mathrm{O} \leftrightarrow \mathrm{NCO}+\mathrm{H}$ & $\mathrm{H}+\mathrm{N}_{2} \mathrm{O} \leftrightarrow \mathrm{NO}+\mathrm{NH}$ \\
$\mathrm{N}+\mathrm{OH} \leftrightarrow \mathrm{NO}+\mathrm{H}$ & $\mathrm{NCO}+\mathrm{H} \leftrightarrow \mathrm{NH}+\mathrm{CO}$ & $\mathrm{O}+\mathrm{N}_{2} \mathrm{O} \leftrightarrow 2 \mathrm{NO}$ \\
& $\mathrm{NH}+\mathrm{H} \leftrightarrow \mathrm{N}+\mathrm{H}_{2}$ & \\
& $\mathrm{~N}+\mathrm{OH} \leftrightarrow \mathrm{NO}+\mathrm{H}$ & \\
\hline
\end{tabular}


$\mathrm{H}_{2} \mathrm{O}+\mathrm{H} \stackrel{k_{6}}{\longrightarrow} \mathrm{OH}+\mathrm{H}_{2}$

$\mathrm{H}_{2}+\mathrm{O} \stackrel{k_{7}}{\longrightarrow} \mathrm{OH}+\mathrm{H}$

$\mathrm{CO}+\mathrm{OH} \stackrel{k_{8}}{\longrightarrow} \mathrm{CO}_{2}+\mathrm{H}$

$\mathrm{O}$ NO ativo é formado pelo mecanismo de radicais derivados do combustível que reagem com o $\mathrm{N}_{2}$ do ar. De uma forma geral, a contribuição do NO ativo para o NO total é pequena para queimadores estacionários, contudo a sua concentração é significante nos turbilhões das chamas ricas, turbulentas e não prémisturadas $^{12}$.

\section{METODOLOGIA PARA PESQUISA COMPUTACIONAL}

Investigou-se o efeito do ar pré-aquecido de combustão na temperatura da chama e, conseqüentemente, na emissão de $\mathrm{NO}_{\mathrm{x}}$ para o queimador Stordy. O efeito da variação da concentração de $\mathrm{N}_{2}$ na composição do gás natural também foi investigado.

Para comparar efetivamente os resultados dos diferentes casos foram mantidos constantes o fluxo de ar e o fluxo de combustível, pois na prática, usuários de gás natural não podem variar as condições de operação com base na composição do gás por falta desta informação.

A temperatura do ar de combustão foi variada entre 300 e 1000 $\mathrm{K}$ considerando no modelo a densidade do ar. Na prática, os equipamentos são ajustados para temperaturas variáveis, pois utilizam o calor remanescente dos gases de combustão para aquecer o ar de entrada. Duas composições de gás natural foram utilizadas para investigar o efeito da concentração de $\mathrm{N}_{2}$ na emissão de $\mathrm{NO}_{\mathrm{x}}$. As composições dos casos investigados são mostradas na Tabela 3.

Os casos 1 e 4 foram realizados com a temperatura do ar a 300 $\mathrm{K}$. Nos casos 2 e 5 a temperatura de pré-aquecimento foi fixada a $600 \mathrm{~K}$ e nos casos 3 e 6, a $1000 \mathrm{~K}$. Os fluxos de gás natural e do ar variaram de acordo com a composição apresentada na Tabela 3, onde o ar foi mantido fixo com $10 \% \mathrm{v}$ de excesso e sofreu apenas variações de temperatura. A velocidade do gás natural variou conforme sua composição, contudo essa variação foi pequena quando comparada com a velocidade máxima da mistura.

\section{RESULTADOS E DISCUSSÃO}

A principal diferença entre os valores medidos para a temperatura e os valores calculados pela simulação em CFD para o sistema fornalha-queimador foi a exclusão da base resfriada à água no modelo computacional. Para se reduzir o número total de células na malha computacional, desprezou-se a base da fornalha e utilizou-se simetricamente apenas um quarto do domínio.

O perfil de temperatura foi calculado ao longo da fornalha, sob condições de operação apresentadas na Tabela 3 (caso 1) e mostra- do na Figura 4. A figura mostra uma temperatura máxima calculada de 1921 K para um ar de combustão a 300 K. Para este ar préaquecido a $600 \mathrm{~K}$ obteve-se $2169 \mathrm{~K}$ e a $1000 \mathrm{~K}, 2293 \mathrm{~K}$ (limite máximo da temperatura de ar pré-aquecido para esse tipo de queimador).

Resultados similares foram obtidos quando o queimador foi operado com uma composição do gás natural com $10 \% \mathrm{v} \mathrm{N} \mathrm{N}_{2}$. A temperatura máxima calculada foi de 1904, 2092 e $2187 \mathrm{~K}$ para as temperaturas do ar de combustão a 300, 600 e 1000 K, respectivamente.

$\mathrm{O} \mathrm{NO}$ ativo e térmico foram calculados e mostrados para o caso 1 (Figuras 5 e 6). O NO combustível não foi considerado no modelo pelo fato do gás natural não conter "nitrogênio orgânico", ou seja, átomos de nitrogênio em sua estrutura molecular. Assim, o NO calculado através dos mecanismos ativo e térmico representa o

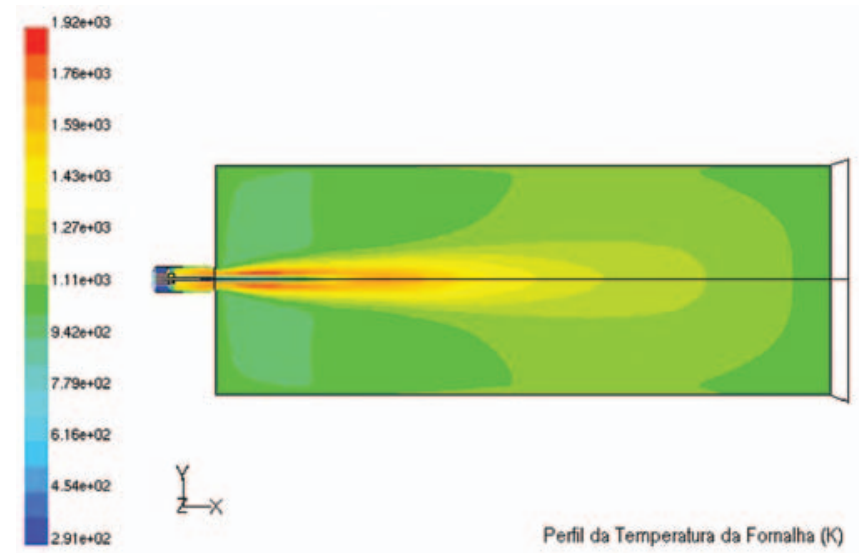

Figura 4. Perfil de temperatura na fornalha para o caso 1

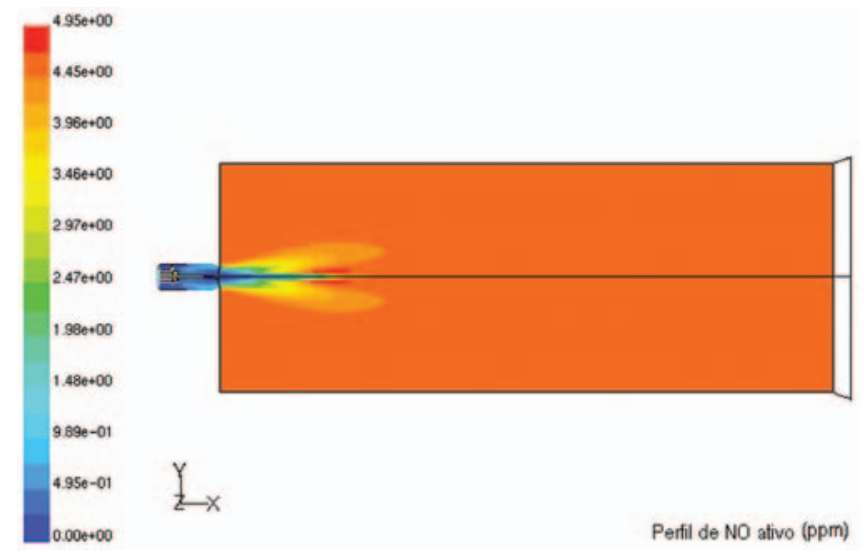

Figura 5. Perfil de concentração de NO ativo para o caso 1

Tabela 3. Parâmetros de combustão para os casos investigados

\begin{tabular}{lccccc}
\hline $\mathrm{Nr}$ & $\begin{array}{c}\mathrm{N}_{2} \text { no Gás } \\
\text { Natural }(\%)\end{array}$ & $\begin{array}{c}\text { Potência } \\
\text { Térmica }(\mathrm{kW})\end{array}$ & $\begin{array}{c}\text { Temperatura } \\
\text { do Ar }(\mathrm{K})\end{array}$ & $\begin{array}{c}\text { (A/F)/ (A/F) esteq. } \\
\text { Velocidade do Gás } \\
\text { na Entrada }\left(\mathrm{m} \mathrm{s}^{-1}\right)\end{array}$ \\
\hline 1 & 1 & 75 & 300 & 1,100 & 12,3 \\
2 & 1 & 75 & 600 & 1,100 & 1,3 \\
3 & 1 & 75 & 1000 & 1,100 & 12,3 \\
4 & 10 & 69 & 300 & 1,187 & 27,4 \\
6 & 10 & 69 & 600 & 1,187 & 27,4 \\
6
\end{tabular}




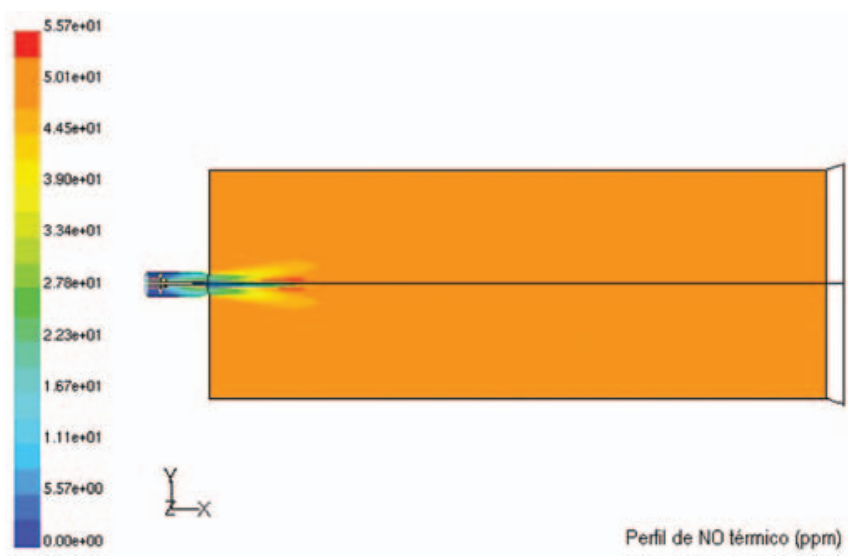

Figura 6. Perfil de concentração de NO térmico para o caso 1

$\mathrm{NO}_{\mathrm{x}}$, pois o modelo empregado é limitado por não considerar o mecanismo de formação de $\mathrm{NO}_{2}$, formado principalmente em zonas de baixas temperaturas.

As concentrações de $\mathrm{NO}$ ativo foram calculadas quando o queimador foi operado sob as condições apresentadas na Tabela 4 para o caso 1. A concentração de NO ativo calculada foi sempre inferior à concentração de NO térmico. O NO ativo foi formado na zona rica em combustível, embora altas concentrações foram também encontradas na região da chama.

Para os casos listados na Tabela 4, o valor máximo para a concentração de NO ativo (27 ppm para o caso 4) foi encontrado próximo ao queimador, na zona de reação. Isso foi devido à existência de uma alta concentração de radicais derivados do combustível, como $\mathrm{CH}$ e $\mathrm{CH}_{2}$, presentes no mecanismo de reação do $\mathrm{NO}$ ativo. $\mathrm{O}$ NO térmico apresentou baixa variação para os casos com $10 \% \mathrm{v}$ de $\mathrm{N}_{2}$ no gás natural. Devido à perda térmica com o aumento de $\mathrm{N}_{2}$, os valores de emissão de $\mathrm{NO}$ para os casos 4, 5 e 6 foram menores que para os casos com $1 \% \mathrm{v}$ de $\mathrm{N}_{2}(1,2$ e 3$)$.

Os resultados das simulações para os casos da Tabela 4 apresentaram níveis de diluição diferentes nos gases de exaustão. As concentrações de $\mathrm{NO}$ foram corrigidas para $0 \% \mathrm{v}$ de $\mathrm{O}_{2}$ em base seca, pois houve necessidade de se considerar a remoção do efeito causado pela diluição dos gases, permitindo uma comparação precisa entre os casos.

A Figura 7 confirma que o aumento do NO térmico é dependente da temperatura de pré-aquecimento do ar de combustão, independentemente do percentual de $\mathrm{N}_{2}$ no gás natural.

O nível de emissão de NO para esse tipo de queimador produziu 99 ppm quando o queimador foi operado com gás natural a $1 \% \mathrm{v} \mathrm{N}_{2}$ e à temperatura do ar na entrada de $300 \mathrm{~K}$. Contudo, o nível de emissão de NO térmico aumentou para 287 ppm quando o ar foi pré-aquecido a $1000 \mathrm{~K}$, superando os níveis de emissão máximos estabelecidos para o gás natural pela legislação ambiental. Resultados similares foram obtidos quando o queimador foi operado com gás natural com $10 \% \mathrm{v} \mathrm{N}_{2}$. O nível de emissão do $\mathrm{NO}$ foi de 82 ppm para uma condição com o ar de combustão a $300 \mathrm{~K}$, aumentando para $233 \mathrm{ppm}$ com ar pré-aquecido a $1000 \mathrm{~K}$. O efeito do aumento da concentração de $\mathrm{N}_{2}$ no gás natural foi verificado comparando-se os níveis de emissão do NO térmico, $\mathrm{NO}$ ativo e NO total dos casos 1,2 e 3 com os casos 4, 5 e 6 .

$\mathrm{O}$ NO total emitido para este tipo de queimador foi reduzido em cerca de $21 \%$ quando a concentração de $\mathrm{N}_{2}$ no gás natural au-

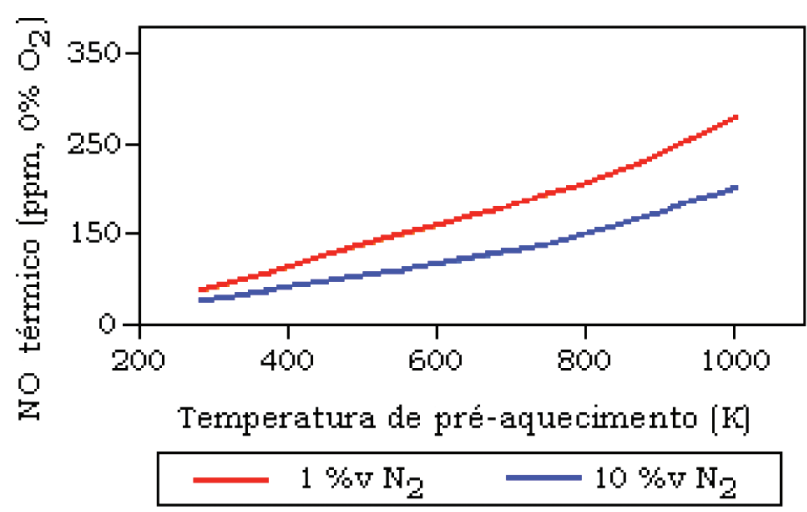

Figura 7. Efeito da temperatura de pré-aquecimento do ar de combustão na emissão de NO térmico

Tabela 4. Valores de temperatura e concentrações das espécies químicas na exaustão para os casos investigados em CFD

\begin{tabular}{|c|c|c|c|c|c|c|}
\hline & $300 \mathrm{~K}$ & $\begin{array}{c}1 \% \mathrm{v} \mathrm{N}_{2} \\
600 \mathrm{~K}\end{array}$ & $1000 \mathrm{~K}$ & $300 \mathrm{~K}$ & $\begin{array}{c}10 \% \mathrm{v} \mathrm{N}_{2} \\
600 \mathrm{~K}^{2}\end{array}$ & $1000 \mathrm{~K}$ \\
\hline Temperatura Máxima (K) & 1858 & 1984 & 2058 & 1845 & 1990 & 2070 \\
\hline Temperatura do Gás de Exaustão (K) & 1640 & 1707 & 1782 & 1584 & 1667 & 1743 \\
\hline \multicolumn{7}{|c|}{ Concentrações das Espécies no Gás de Exaustão } \\
\hline $\mathrm{O}_{2}(\% \mathrm{v})$ & 1,7 & 2,0 & 2,0 & 3,0 & 3,2 & 3,2 \\
\hline $\mathrm{CO}_{2}(\% \mathrm{v})$ & 9,0 & 8,9 & 8,9 & 8,4 & 8,3 & 8,3 \\
\hline $\mathrm{CO}^{2}(\mathrm{ppm})$ & 0 & 0 & 0 & 0 & 0 & 0 \\
\hline $\mathrm{H}_{2} \mathrm{O}(\% \mathrm{v})$ & 17,3 & 17,1 & 17,0 & 16,0 & 15,8 & 15,8 \\
\hline $\mathrm{N}_{2}(\% \mathrm{v})$ & 71,8 & 72,0 & 72,0 & 72,5 & 72,6 & 72,6 \\
\hline NO ativo (ppm) & 4 & 5 & 6 & 19 & 22 & 26 \\
\hline NO ativo $*$ & 5 & 6 & 7 & 21 & 24 & 29 \\
\hline NO térmico (ppm) & 89 & 157 & 264 & 56 & 108 & 190 \\
\hline NO térmico $*$ & 94 & 166 & 280 & 61 & 117 & 204 \\
\hline NO total * & 99 & 172 & 287 & 82 & 141 & 233 \\
\hline$\%$ NO ativo & 5 & 3 & 2 & 26 & 17 & 12 \\
\hline$\%$ NO térmico & 95 & 97 & 98 & 74 & 83 & 88 \\
\hline
\end{tabular}

* Corrigido para $0 \% \mathrm{O}_{2}$ 
mentou de 1 para $10 \%$ v quando utilizado ar de combustão à temperatura de $300 \mathrm{~K}$, e em cerca de $23 \%$ para uma temperatura do ar de combustão a $1000 \mathrm{~K}$. Contudo, a contribuição do NO ativo aumentou de 5 para $26 \%$ quando a concentração de $\mathrm{N}_{2}$ no gás natural aumentou de 1 para $10 \% \mathrm{v}$ para o ar de combustão à temperatura a $300 \mathrm{~K}$ e de 2 para $12 \%$ para ar pré-aquecido à temperatura de 1000 $\mathrm{K}$. Com o aumento da quantidade de $\mathrm{N}_{2}$ no gás natural, a temperatura do gás de exaustão foi reduzida além da zona de reação. Assim, uma chama mais curta foi obtida para o caso com maior concentração de $\mathrm{N}_{2}$ no gás natural e, conseqüentemente, maior destaque ao mecanismo ativo com espécies intermediárias.

A Figura 8 mostra os efeitos do ar pré-aquecido de combustão e da concentração de $\mathrm{N}_{2}$ no gás natural em relação ao NO térmico para o queimador utilizado. O gráfico mostra que, a temperaturas de pré-aquecimento menores que $1000 \mathrm{~K}$, a temperatura do ar préaquecido apresentou um efeito mínimo na emissão do NO térmico quando comparado com o seu efeito a temperaturas de pré-aquecimento mais elevadas que $1000 \mathrm{~K}$. Assim, uma atenção especial deve ser dada à emissão de NO quando o ar de combustão for préaquecido a uma temperatura próxima de $1000 \mathrm{~K}$ ou eventualmente maior.

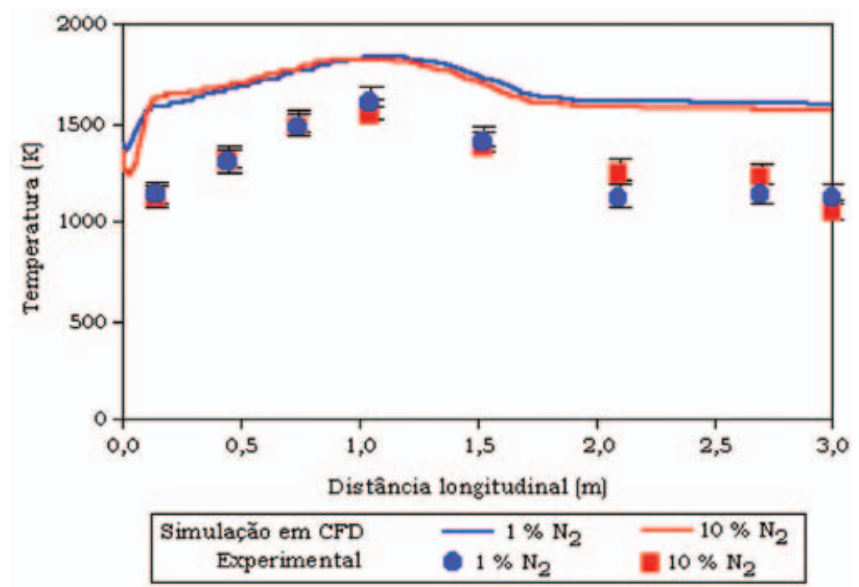

Figura 8. Temperatura do gás de combustão ao longo do eixo central da fornalha

A formação do NO térmico tem grande dependência com a temperatura e isso foi verificado através do cálculo da taxa de formação de NO térmico pela Equação 4. A taxa de formação do NO térmico para o caso 3 foi a mais alta calculada, $1,3310^{-4} \mathrm{~kg} \mathrm{~m}^{-3} \mathrm{~s}^{-1}$, com base na temperatura máxima dentro da fornalha.

O pré-aquecimento do ar de combustão é um dos modos mais tradicionais para se produzir chamas a altas temperaturas, mas os níveis de emissão de NO devem ser monitorados na aplicação desta técnica.

Para os experimentos realizados com o queimador Stordy, o ar de combustão foi introduzido na zona de combustão em dois estágios (externo principal e central secundário) para reduzir as emissões de $\mathrm{NO}_{\mathrm{X}}$.

Os resultados obtidos pelas simulações computacionais para os cálculos do NO térmico e NO ativo mostraram que a contribuição do NO ativo foi menor que a do NO térmico quando o ar de combustão foi pré-aquecido. Contudo, a contribuição do NO ativo aumentou quando o queimador foi operado com maior percentual de $\mathrm{N}_{2}$ do gás natural.

Os resultados de CFD confirmaram que a oxidação do nitrogênio atmosférico $\left(\mathrm{N}_{2}\right)$ pelo mecanismo térmico foi a principal rota para emissão de $\mathrm{NO}_{\mathrm{x}}$ para este tipo de queimador.
A Figura 9 mostra que o modelo computacional subestimou a quantidade de $\mathrm{NO}_{\mathrm{x}}$ produzido dentro da fornalha, que foi medido através de uma sonda isocinética em diferentes distâncias em relação à posição do queimador. Contudo, os perfis de concentração dos modelos computacional e experimental mostraram boa correlação sobre o efeito da variação da concentração de $\mathrm{N}_{2}$ no gás natural para temperatura e $\mathrm{NO}_{\mathrm{x}}$ apresentadas nas Figuras 7 e 8.

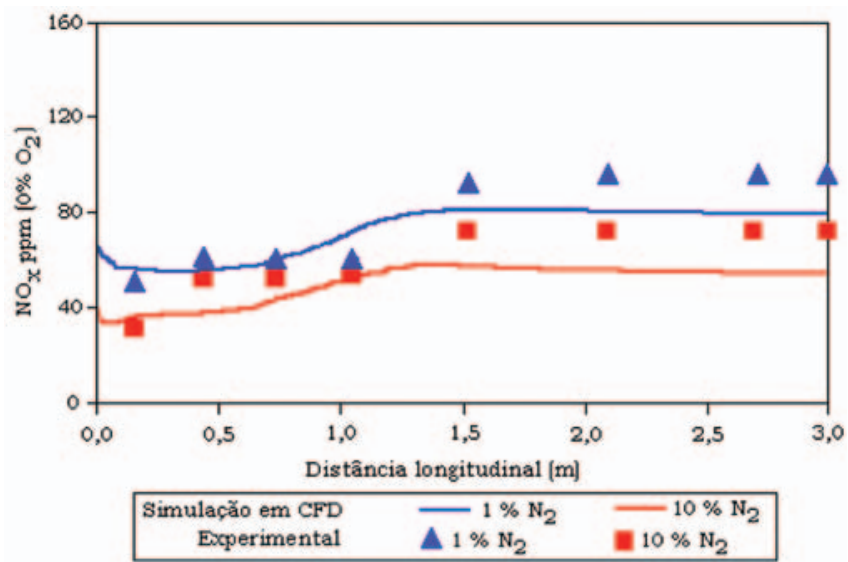

Figura 9. Concentração de $\mathrm{NO}_{\mathrm{X}}$ ao longo do eixo central da fornalha

Neste trabalho foram encontradas pequenas discrepâncias entre os resultados experimentais e computacionais, permitindo certificar a validação do modelo CFD empregado. Os resultados obtidos do modelo CFD mostraram que o NO ativo teve uma contribuição significante no NO total emitido para queimadores de ar estagiado. A importância do NO ativo diminuiu com o aumento da temperatura do ar de combustão.

Os resultados de CFD também mostraram que, ao se utilizar temperaturas de pré-aquecimento do ar de combustão próximas a $1000 \mathrm{~K}$, deve-se estimar altos níveis de emissões de $\mathrm{NO}_{\mathrm{x}}$ para o processo.

\section{CONCLUSÕES}

Investigações teóricas usando CFD com uma integração de dados experimentais mostraram que o aumento da concentração de $\mathrm{N}_{2}$ no gás natural provocou diversos efeitos em seu processo de combustão. Entre eles, redução da temperatura máxima da chama, menor emissão total de $\mathrm{NO}_{\mathrm{x}}$, redução na concentração máxima de $\mathrm{O}$ atômico e $\mathrm{OH}$ na zona de combustão, redução da transferência de calor da chama para a parede da fornalha causada pela perda de energia, absorvida pelas reações de recombinação das espécies instáveis $\mathrm{O}, \mathrm{H}, \mathrm{OH}$ e $\mathrm{HO}_{2}$. Assim, do ponto de vista ambiental, a alta concentração de $\mathrm{N}_{2}$ no gás natural é positiva, pois a emissão de $\mathrm{NO}_{\mathrm{x}}$ será sempre menor. A menor capacidade calorífica do combustível com maior teor de nitrogênio diminui a temperatura de pico da chama, reduz as concentrações das espécies $\mathrm{O}$ atômico e $\mathrm{OH}$ e, conseqüentemente, a formação do NO térmico, maior responsável pelo NO total.

Os resultados experimentais mostraram que a emissão de $\mathrm{NO}_{\mathrm{x}}$ foi reduzida em $21 \%$ quando a concentração de $\mathrm{N}_{2}$ no gás natural aumentou de 1 para $10 \% \mathrm{v}$ para um ar de combustão a temperatura de $300 \mathrm{~K}$. O aumento da concentração de $\mathrm{N}_{2}$ de 1 para $10 \% \mathrm{v}$ no ar de combustão pré-aquecido à temperaturas de 600 e $1000 \mathrm{~K}$ (investigadas computacionalmente) produziu uma redução de 22 e $23 \%$ na emissão de $\mathrm{NO}_{\mathrm{x}}$. A desvantagem associada ao aumento da concentração de $\mathrm{N}_{2}$ no gás natural foi a redução da transferência de calor da chama para a parede da fornalha. Essa conclusão respalda 
o aspecto econômico, pois diminui a eficiência do combustível. $\mathrm{Na}$ prática, o consumidor de gás precisará queimar maior quantidade de combustível para obter a mesma eficiência térmica quando o gás natural apresentar uma concentração de nitrogênio mais elevada.

Investigações experimentais e modelagem com CFD confirmaram que a formação do NO térmico é fortemente dependente da temperatura de chama. Com a modelagem CFD foi possível relacionar o ar de combustão pré-aquecido, a temperatura de chama e o NO térmico formado. Os resultados mostraram que o efeito do aumento do ar pré-aquecido nas emissões de $\mathrm{NO}_{\mathrm{x}}$ foi mais significativo quando o ar foi pré-aquecido a temperaturas próximas a 1000 K. Assim, para sistemas de combustão que demandam alta eficiência térmica com temperaturas de chama elevadas no interior da fornalha maiores que $2000 \mathrm{~K}$, técnicas adicionais de redução de $\mathrm{NO}_{\mathrm{x}}$ devem ser utilizadas, como por ex., a recirculação de gases de exaustão (FGR).

A modelagem em CFD indicou o mecanismo térmico como a principal rota para formação de NO sob temperaturas de chama maiores que $2000 \mathrm{~K}$. A modelagem em CFD mostrou que a contribuição máxima do $\mathrm{NO}$ ativo para a formação total de $\mathrm{NO}_{\mathrm{x}}$, calculada para a exaustão, foi de $26 \%$ com o ar de combustão à temperatura de $300 \mathrm{~K}$ e concentração de $\mathrm{N}_{2}$ no gás natural de $10 \% \mathrm{v}$ (caso 4). Embora a concentração absoluta de NO ativo tenha sido aumentada proporcionalmente com pré-aquecimento do ar de combustão, a sua contribuição para a emissão total de $\mathrm{NO}_{\mathrm{X}}$ no caso do sistema queimador-fornalha investigado foi reduzida com o aumento do ar pré-aquecido de combustão. Assim, constatou-se que o NO ativo não pode ser negligenciado nos sistemas de combustão, pois sua contribuição é significativa nas emissões totais de $\mathrm{NO}_{\mathrm{x}}$ para queimadores de ar estagiado. Contudo, a importância do NO ativo diminui quando a temperatura do ar de combustão aumenta. Isto se deve à maior dependência do $\mathrm{NO}_{\mathrm{x}}$ total com o mecanismo térmico, onde a temperatura assume o papel de agente principal.

Uma comparação entre os resultados experimentais e o modelo em CFD mostrou boa concordância entre as duas fontes de da- dos. Em geral, modelos em CFD para engenharia de combustão devem ser validados com resultados experimentais, pois a modelagem é uma ferramenta útil para a pesquisa aplicada. Seu uso é recomendado para pesquisas com sistemas de difícil controle, com substancial redução no custo e no tempo de execução.

\section{AGRADECIMENTOS}

À CAPES pela bolsa de doutoramento de L. P. Rangel, ao Departamento de Combustíveis e Energia da Universidade de Leeds (Reino Unido) pelo suporte laboratorial e computacional na realização deste trabalho.

\section{REFERÊNCIAS}

1. Ministério de Minas e Energia do Brasil (MME); Balanço Energético Doméstico, 2004.

2. Agênica de Proteção Ambiental dos Estados Unidos (EPA-US); Nitrogen Oxide Control of Stationary Combustion Sources, 2000.

3. Missaghi, M.; Pourkashanian, M.; Williams, A.; Yap, L.; The Prediction of NO Emission From an Industrial Burner, American Flame Day: San Francisco, CA, 1991.

4. Geerssen, T. M.; Physical Properties of Natural Gases, N. V. Nederlese Gasunie, 1988

5. Fluent User's Guide; Fluent Inc.; Lebanon - NH, 1998.

6. Versteeg, H. K.; Malalasekera, W.; An Introduction to Computational Fluid Dynamics, Longman Group, 1995, p. 3.

7. Yap, L.; Pourkashanian, M.; Williams, A.; Modelling of Natural Gas Combustion with Oxygen e Preheated Air, BFRC Flame Days, 1994.

8. Launder, B. E.; Spalding, D. B.; Lectures in Mathematical Models of Turbulence, Academic Press; London, 1972.

9. Turns, S. R.; An Introduction to Combustion: Concepts and Applications, Ed. McGraw-Hill, 1996, p. 199.

10. Tomeczek, J.; Goral, J.; Gradon, B.; 25 th Symposium International on Combustion, The Combustion Institute, 1994.

11. Bartok, W.; Sarofim, A. F.; Fossil Fuel Combustion, John Wiley \& Sons Inc., 1989.

12. Miller, J. A.; Bowman, C. T.; Progress on Engineering Combustion Science, 1989, vol. 15, p. 287. 